\title{
Adaptive Forwarding Strategy Based on MCDM Model in Named Data Networking
}

\author{
Cong $\mathrm{Pu}$ \\ Department of Computer Sciences and Electrical Engineering \\ Marshall University, Huntington, WV 25755 \\ Email: puc@marshall.edu
}

\begin{abstract}
Named Data Networking (NDN), as a specific architecture design of Information-Centric Networking (ICN), has quickly became a promising candidate for future Internet architecture, where communications are driven by data names instead of IP addresses. To realize the NDN architecture in the future Internet, a stateful forwarding plane has been proposed to maintain the pending Interest packets and guide Data packets back to the consumers. However, the operations of stateful forwarding plane are not fully explained in NDN project and the design specifics remain to be filled in. In addition, the overall framework of stateful forwarding plane should be adaptive and responsive to diverse network conditions by taking into account of multiple network metrics. In this paper, we propose a novel adaptive forwarding strategy, also referred to as $f w d^{P R O}$, to realize intelligent and adaptive Interest packet forwarding in NDN. The basic idea of the $f w d^{P R O}$ is to employ Technique for Order Performance by Similarity to Idea Solution (TOPSIS) to dynamically evaluate outgoing interface alternatives based on multiple network metrics and objectively select an optimal outgoing interface to forward the Interest packet. The TOPSIS is a multi-criteria decision-making (MCDM) model to identify the best alternative that is nearest to the positive ideal solution and farthest from the negative ideal solution. We conduct extensive simulation experiments for performance evaluation and comparison with the existing BestRoute and EPF schemes. The simulation results show that the proposed adaptive forwarding strategy can improve the Interest satisfaction ratio and Interest satisfaction latency as well as reduce the average hop count.
\end{abstract}

Index Terms-Named Data Networking, Adaptive Forwarding Strategy, Multi-Criteria Decision-Making Model, TOPSIS

\section{INTRODUCTION}

Over the last decade, the number of devices connected to the Internet has been rapidly increasing due to the proliferation of emerging technologies such as Internet of Things, Artificial Intelligence, and Blockchain [1], [2]. According to Cisco Annual Internet Report [3], there will be 29.3 billion networked devices by 2023. Even though the global network performance will be significantly improved, e.g., the fixed broadband speed and mobile network connection speed will reach 110.4 Mbps and 43.9 Mbps in 2023 respectively, the fast growth of connected devices still put high pressure on the underlying Internet infrastructure which was developed in the 1970s. Today's Internet has exceeded all expectations for facilitating conversations between communication endpoints but shows signs of aging when it meets with next generation content-oriented services and applications [4]. Thus, in order to keep pace with a changing world, a future Internet architecture, Named Data Networking [5], has been regarded as the

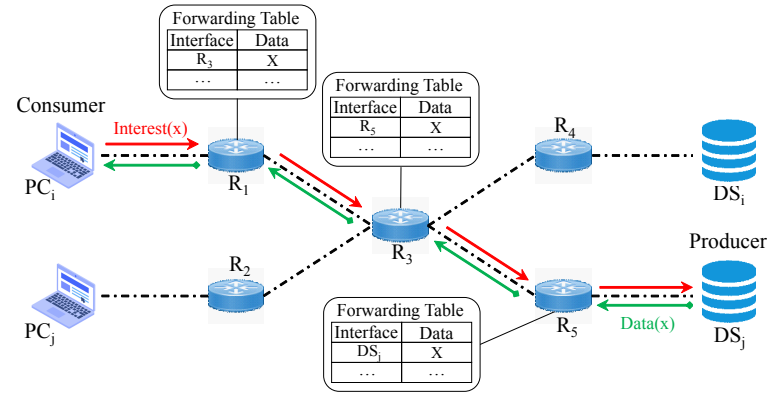

Fig. 1. NDN communication model.

most promising Internet architecture to drive further growth and success of the future Internet.

In NDN, all communications are performed by using Interest and Data packets, both of which carry data (or content) names rather than host (or physical location) addresses. The data names are hierarchically structured like URLs, e.g., the first segment of author's paper may have the name /marshall.edu/cs/congpu/papers/ndn2020.pdf/segment1, where ' $/$ delineates name components in text representations. This hierarchical structure allows applications to represent the context and relationships of data elements and facilitate traffic demultiplexing. As shown in Fig. 1, to retrieve data, data consumer (e.g., $P C_{i}$ ) first sends out an Interest packet piggybacked with the name of desired data (i.e., $x$ ). When a router (e.g., $R_{1}$ ) receives the Interest packet, it forwards the Interest packet toward the data producer(s) based on the information in the forwarding table. Along the forwarding path, any router (e.g., $R_{1}, R_{3}$, or $R_{5}$ ) or data producer (e.g., $D S_{j}$ ) who has the requested data can send back a Data packet piggybacked with the requested data along the reverse path of the Interest packet.

Designing and evaluating the adaptive forwarding plane have been a major challenge within the overall NDN research area [6]. Since NDN was proposed in 2010, there have been many research efforts focusing on this challenge and a rich literature has been developed. The authors in [7] describe an initial design of NDN's forwarding plane and evaluate its data delivery performance under adverse network conditions. Unquestionably, [7] stands out as one of the notable landmarks that sketches a basic picture of NDN's forwarding plane which includes data delivery performance assessment, network load management, and Interest retransmission. However, [7] and its future variants fail to consider multiple network metrics when 
accessing the status of outgoing interfaces, which causes the forwarding strategy not to be adaptive and sensitive to network condition changes.

In this paper, we propose a novel adaptive forwarding strategy endowed with good extensibility and flexibility to achieve the goal of intelligent and adaptive Interest packet forwarding in NDN and measure its performance through extensive simulation experiments. Our major contribution is briefly summarized in twofold:

1) We propose a novel adaptive forwarding strategy, also referred to as $f w d^{P R O}$, to realize intelligent and adaptive Interest packet forwarding in NDN. The $f w d^{P R O}$ employs Technique for Order Performance by Similarity to Idea Solution (TOPSIS) to dynamically evaluate outgoing interface alternatives based on multiple network metrics and objectively select an optimal outgoing interface to forward the Interest packet.

2) We design $f w d^{P R O}$ with the consideration of multiple network metrics to respond to network condition changes accurately and astutely. In addition, the $f w d^{P R O}$ offers good flexibility and extensibility. Thus, additional network metrics can be easily included in the forwarding strategy. We revisit prior forwarding strategies, BestRoute [7] and EPF [8], and modify them to work in the framework for performance comparison.

We develop a customized discrete event-driven simulation framework using OMNeT++ [9] and evaluate its performance through extensive simulation experiments in terms of Interest satisfaction ratio, Interest satisfaction latency, and average hop count. The simulation results indicate that the proposed adaptive forwarding strategy $f w d^{P R O}$ can improve Interest satisfaction ratio and Interest satisfaction latency as well as reduce average hop count, indicating a viable forwarding strategy in NDN.

The rest of the paper is organized as follows. Prior forwarding strategies are presented and analyzed in Section II. In Section III, the basic operations of NDN's stateful forwarding plane are summarized. The proposed adaptive forwarding strategy is presented in Section IV. Section V focuses on simulation results and their analyses. Finally, concluding remarks and future research directions are provided in Section VI.

\section{RELATED WORK}

In this section, we present and analyze a variety of up-todate forwarding strategies in NDN.

The authors in [10] propose a cooperative forwarding strategy for NDN networks, where routers share their information such as data names and interfaces to optimize their packet forwarding decisions and estimate the probability of each downstream path to swiftly retrieve the requested data. However, each router needs to collect the information about the names of data being exchanged from neighboring routers, which generates a large number of control messages, and in turn increases the communication overhead in NDN networks. In [11], a forwarding strategy is proposed to balance the tradeoff between network overhead and performance satisfactory in
Internet of Things environments. Each node overhears Data packets and learns a cost value by reinforcement, and then decides to broadcast an Interest packet with a delay according to their cost-based eligibility. The broadcast-based forwarding on the top of MAC layer can reduce communication overhead, however, a node might not be able to forward the Interest packet timely if the wireless medium is always busy. The authors in [12] propose a forwarding strategy named IFS-RL based on reinforcement learning. The IFS-RL trains a neural network model which chooses appropriate interfaces for the forwarding of Interest based on observations of the resulting performance of past decisions collected by routing node. The IFS-RL can achieve the goal of improving throughput and packet drop rate, but fails in load balancing.

In [13], a forwarding strategy is proposed for persistent Interests in NDN, where forwarding decisions are based on a combination of information from the forwarding information base and probing results. Clients issue probing Interests in order to rate paths through the network, and all probe-receiving routers can use them to evaluate the performance of already known paths, but also to explore new, possibly better paths. Nevertheless, the probing Interest packets will significantly increase network traffic and cause other issues such as traffic congestion and packet loss. The authors in [14] exploit the partially observable Markov decision process (POMDP) to design NDN request forwarding mechanism based upon the key concept of event. Since the exact optimal solution of POMDP problems in general is extremely computationally demanding, a simulation-based optimization algorithm is also proposed to find the approximate optimal solution. In [15], a deep reinforcement learning based forwarding strategy is proposed in NDN, where the information such as data content, interface status, and network states are first collected during the forwarding process. After that, the collected information is used as input of the deep reinforcement learning for training, whose result will be used as the forwarding strategy to guide the forwarding of Interest packets. The [6] provides a list of requirements of NDN forwarding plane and compares all available schemes proposed for NDN forwarding plane based on the data structure utilized. In addition, this survey paper discusses some issues, challenges, and directions in future research.

In summary, most prior forwarding strategies rely on machine learning techniques or probability theory to make Interest packet forwarding decision. However, little attention has been paid to an adaptive forwarding strategy based on multicriteria decision-making model in NDN, where Technique for Order Performance by Similarity to Idea Solution (TOPSIS) is used to dynamically evaluate outgoing interface alternatives based on multiple network metrics and objectively select an optimal outgoing interface to forward the Interest packet. Another desirable feature is that the proposed adaptive forwarding strategy is designed with the consideration of extensibility and flexibility. Therefore, additional network metrics can be easily added in the forwarding plane in order to adapt to the changes in network conditions. 


\section{Overview of NDN Stateful ForWARding Plane}

\section{A. Processing of Interest and Data Packets}

In NDN, a data consumer can retrieve data by issuing an Interest packet piggybacked with the name of desired data to the network. When a router receives the Interest packet, it first checks whether its Content Store already caches the desired data or not. Here, router's Content Store is a temporary cache of Data packets it has received. If the desired data exists in the Content Store, the router replies a Data packet piggybacked with the desired data back to the consumer along the reverse path of Interest packet. Otherwise, the router checks the name of desired data with each entry in the Pending Interest Table. In NDN, the Pending Interest Table stores the forwarded Interest packets but have not been satisfied by Data packets yet. In addition, each Pending Interest Table entry contains four components: data name, nonce, incoming interface of the Interest packet has been received from, and outgoing interface of the Interest packet has been forwarded to. If the Pending Interest Table contains an entry with the same data name and nonce as the Interest packet, the router immediately drops the Interest packet because the Interest packet that has been forwarded before is looped back. If there is en entry with matching data name and unmatching nonce, the router just adds a new entry with data name, nonce, and incoming interface without forwarding the Interest packet since this Interest packet is considered as subsequent Interest. If the data name and nonce do not match with any entry in the Pending Interest Table, the router forwards the Interest packet to an outgoing interface according to forwarding strategy, and adds a new entry in the Pending Interest Table.

When the Interest packet reaches the data producer or the router who caches the desired data in the Content Store, a Data packet piggybacked with the desired data is replied back to the consumer. When a router receives the Data packet from an upstream router or the producer, it first searches the piggybacked data name in the Pending Interest Table. If an entry with matching data name is found in the Pending Interest Table, the router forwards the Data packet to all stored incoming interfaces, caches a copy of piggybacked data in the Content Store, and removes all entries with matching data name from the Pending Interest Table. Otherwise, the router drops the Data packet because the data is unsolicited. In order to purge the stale entry in the Pending Interest Table, an entry lifetime is assigned to each entry. When the lifetime expires, the entry is removed from the Pending Interest Table. The processing of Interest and Data packets are shown in Fig. 2.

\section{B. Interest Packet Forwarding Strategy}

The forwarding strategy makes Interest packet forwarding decision based on the information stored in the Forwarding Information Base, where each entry records a name prefix and a list of outgoing interfaces together with their associated forwarding preference. The forwarding preference reflects forwarding policy as well as the cost of forwarding path which is typically calculated using certain network metrics.

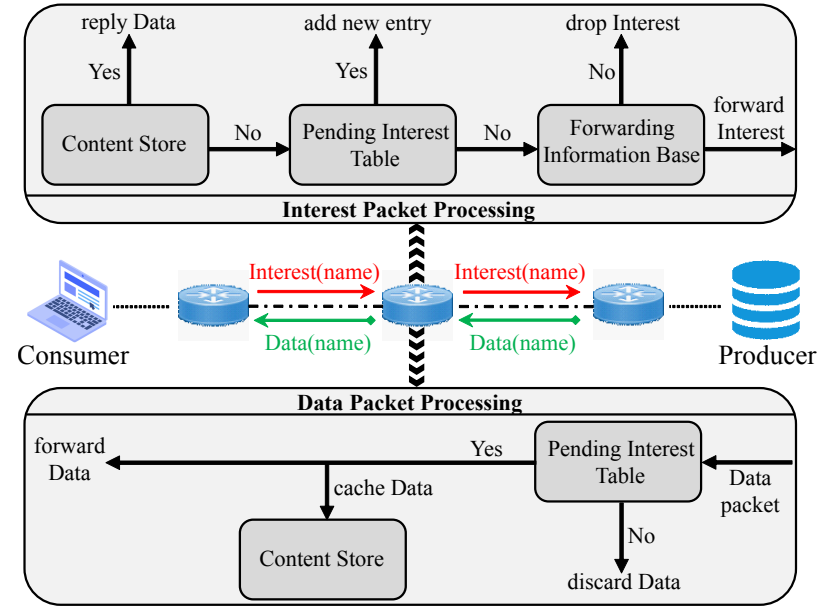

Fig. 2. Processing of Interest and Data packets in NDN.

For example, the BestRoute [7] adopts the coloring scheme to represent the working status of each outgoing interface, based on which the forwarding strategy selects the best outgoing interface to forward an Interest packet. For each name prefix, all outgoing interfaces are ranked based on Interest rate limit and the highest ranked Green outgoing interface is always selected to forward an Interest packet. If there is no Green outgoing interface, the highest ranked Yellow outgoing interface is adopted. The Red outgoing interfaces are never used because they cannot bring data back. The forwarding strategy in the BestRoute can reduce network traffic. However, it fails to detect and respond to network condition changes timely. For instance, if the Interest packet forwarding rate reaches the rate limit, the outgoing interface will experience traffic congestion sooner or later, which leads to the fact that the second-ranked Green outgoing interface would be the best option for Interest packet forwarding. Thus, the highest ranked Green outgoing interface may not always be the best option with various changes in network conditions. In summary, the forwarding strategy is playing an important role in NDN forwarding plane. In order to improve the network performance and respond to network condition changes accurately and astutely, the forwarding strategy should take into account of multiple network metrics to make Interest packet forwarding decision.

\section{The Proposed Adaptive Forwarding Strategy}

The basic idea of the proposed adaptive forwarding strategy $\left(f w d^{P R O}\right)$ is to employ Technique for Order Performance by Similarity to Idea Solution (TOPSIS) to dynamically evaluate outgoing interface alternatives based on multiple network metrics and objectively select an optimal outgoing interface to forward the Interest packet. The TOPSIS is a multi-criteria decision-making (MCDM) model to identify the best alternative that is nearest to the positive ideal solution and farthest from the negative ideal solution [16]. When a router receives the Interest packet to forward, it evaluates all outgoing interface alternatives based on the up-to-date network metrics information and calculates the forwarding index of each outgoing interface. Based on the forwarding index, the 
router ranks all outgoing interface alternatives and selects the highest ranked outgoing interface to forward the Interest packet. The detailed design of the $f w d^{P R O}$ is provided in the following.

First, the router establishes a decision matrix with the up-todate network metrics information for the ranking of outgoing interface alternatives. The structure of the decision matrix can be expressed as follows:

$$
M=\begin{gathered}
A I_{1} \\
A I_{2} \\
\vdots \\
A I_{i} \\
\vdots \\
A I_{m}
\end{gathered}\left[\begin{array}{cccccc}
P M_{1} & P M_{2} & \ldots & P M_{j} & \ldots & P M_{n} \\
x_{11} & x_{12} & \ldots & x_{1 j} & \ldots & x_{1 n} \\
x_{21} & x_{22} & \cdots & x_{2 j} & \ldots & x_{2 n} \\
\vdots & \vdots & \ldots & \vdots & \ldots & \vdots \\
x_{i 1} & x_{i 2} & \cdots & x_{i j} & \ldots & x_{i n} \\
\vdots & \vdots & \ldots & \vdots & \ldots & \vdots \\
x_{m 1} & x_{m 2} & \cdots & x_{m j} & \cdots & x_{m n}
\end{array}\right],
$$

where $A I_{i}$ represents the $i^{\text {th }}$ outgoing interface alternative, $i$ $=1,2, \ldots, m ; P M_{j}$ denotes the $j^{t h}$ network metrics, $j=1$, $2, \ldots, n$; and $x_{i j}$ is a crisp value of the $j^{t h}$ network metrics related to the $i^{\text {th }}$ outgoing interface alternative. Second, the router generates the normalized decision matrix $M^{n o r m}\left(=x_{i j}^{*}\right)$ according to

$$
x_{i j}^{*}=\frac{x_{i j}}{\sqrt{\sum_{j=1}^{n} x_{i j}^{2}}}, \quad i=1,2, \ldots, m ; j=1,2, \ldots, n .
$$

Since the scales of measurement for multiple network metrics are not unique, it is important to normalize the decision matrix to make crisp values comparable to each other. Third, the router calculates the weighted normalized decision matrix by multiplying the normalized decision matrix by the relative weights of multiple network metrics. The weighted normalized decision matrix $M^{w g t}\left(=x_{i j}^{\oplus}\right)$ is calculated as

$$
x_{i j}^{\oplus}=w_{j} \times x_{i j}^{*}, \quad i=1,2, \ldots, m ; j=1,2, \ldots, n .
$$

Here, $w_{j}$ represents the relative weight of the $j^{t h}$ network metrics. The rationale behind the design of $w_{j}$ is to adjust the effect of the $j^{\text {th }}$ network metrics for subjective preference. The relative weights of multiple network metrics can be determined by applying Analytic Network Process (ANP) [16]. Fourth, the router calculates the separation measurement using $m$ dimensional Euclidean distance. The separation between the $i^{t h}$ outgoing interface alternative and positive-ideal solution, denoted as $\mathrm{Sol}_{i}^{+}$, is given as

$$
\text { Sol }_{i}^{+}=\sqrt{\sum_{j=1}^{n}\left(x_{i j}^{\oplus}-\max \left(P M_{j}\right)\right)^{2}}, \quad i=1,2, \ldots, m .
$$

Similarly, the separation between the $i^{t h}$ outgoing interface alternative and negative-ideal solution, denoted as $\mathrm{Sol}_{i}^{-}$, is as follows:

$$
\operatorname{Sol}_{i}^{-}=\sqrt{\sum_{i=1}^{n}\left(x_{i j}^{\oplus}-\min \left(P M_{j}\right)\right)^{2}}, \quad i=1,2, \ldots, m .
$$

Based on the separation measurements, the router can calculate the relative closeness of the $i^{t h}$ outgoing interface alternative to the idea solution as follows

$$
\mathbb{I}_{i}=\frac{S_{o l}^{-}}{S o l_{i}^{-}+S_{i}^{+}}, \quad i=1,2, \ldots, m .
$$

Here, the $\mathbb{I}_{i}$ is considered as the forwarding index of the $i^{t h}$ outgoing interface alternative. The $\mathbb{I}_{i}$ lies between 0 and 1 , and the larger the forwarding index value means the better overall performance of the $i^{\text {th }}$ outgoing interface alternative. Finally, the router ranks the forwarding indexes of all outgoing interface alternatives and the highest ranked outgoing interface will be the optimal one to forward the Interest packet.

For example, suppose that a route has four outgoing interface alternatives $\left(\mathrm{AI}_{1-4}\right)$ to choose and forward the Interest packet. We consider interface utilization ratio, round-trip time (RTT), and NACK ratio as real-time network metrics to calculate the forwarding index of each outgoing interface alternative. The decision matrix containing the crisp value of network metrics is shown in Table I. According to Eq. (1) and (2), the normalized decision matrix and the weighted normalized decision matrix is calculated and presented in Table II and III, respectively. Here, the relative weight of interface utilization ratio, RTT, and NACK ratio is set to $0.3,0.4$, and 0.3 , respectively. After that, the separation measurement between each outgoing interface alternative and the positive and negative ideal solutions can be calculated by using the data in Table III, and related results are shown in Table IV.

TABLE I

DECISION MATRIX FOR INTERFACE ALTERNATIVES

\begin{tabular}{cccc}
\hline \hline Interface ID & Interface Util. Ratio & RTT & NACK Ratio \\
\hline$A I_{1}$ & $75 \%$ & $73 \mathrm{~ms}$ & $25 \%$ \\
$A I_{2}$ & $25 \%$ & $45 \mathrm{~ms}$ & $10 \%$ \\
$A I_{3}$ & $62 \%$ & $67 \mathrm{~ms}$ & $19 \%$ \\
$A I_{4}$ & $84 \%$ & $80 \mathrm{~ms}$ & $30 \%$ \\
\hline
\end{tabular}

TABLE II

NORMALIZED DECISION MATRIX FOR INTERFACE ALTERNATIVES

\begin{tabular}{cccc}
\hline \hline Interface ID & Interface Util. Ratio & RTT & NACK Ratio \\
\hline$A I_{1}$ & 0.69699 & 0.67840 & 0.23233 \\
$A I_{2}$ & 0.47673 & 0.85812 & 0.19069 \\
$A I_{3}$ & 0.66494 & 0.71856 & 0.20377 \\
$A I_{4}$ & 0.70107 & 0.66769 & 0.25038 \\
\hline
\end{tabular}

TABLE III

Weighted Normalized DeCision MATRIX FOR INTERfaCe ALTERNATIVES

\begin{tabular}{cccc}
\hline \hline Interface ID & Interface Util. Ratio & RTT & NACK Ratio \\
\hline$A I_{1}$ & 0.20910 & 0.27136 & 0.06970 \\
$A I_{2}$ & 0.14302 & 0.34325 & 0.05721 \\
$A I_{3}$ & 0.19948 & 0.28742 & 0.06113 \\
$A I_{4}$ & 0.21032 & 0.26708 & 0.07511 \\
\hline
\end{tabular}

In the final ranking stage, by using Eq. (5), the forwarding index of each outgoing interface alternative is calculated. The calculated forwarding indexes are ranked and listed in 
TABLE IV

SeParation Distances For InTERFACE Alternatives

\begin{tabular}{ccc}
\hline \hline Interface ID & $\mathrm{Sol}^{+}$ & $\mathrm{Sol}^{-}$ \\
\hline$A I_{1}$ & 0.07210 & 0.06739 \\
$A I_{2}$ & 0.06964 & 0.07617 \\
$A I_{3}$ & 0.05857 & 0.06014 \\
$A I_{4}$ & 0.07617 & 0.06964 \\
\hline
\end{tabular}

TABLE V

FORWARDING INDEX RANKING FOR INTERFACE ALTERNATIVES

\begin{tabular}{ccc}
\hline \hline Rank & Interface ID & Forwarding Index \\
\hline 1 & $A I_{2}$ & 0.52239 \\
2 & $A I_{3}$ & 0.50661 \\
3 & $A I_{1}$ & 0.48312 \\
4 & $A I_{4}$ & 0.47761 \\
\hline
\end{tabular}

Table V. According to the forwarding index, the ranking order of four outgoing interface alternatives is $A I_{2}, A I_{3}$, $A I_{1}$, and $A I_{4}$, which indicates that $A I_{2}$ is the best outgoing interface candidate to choose and forward Interest packet. Major operations of the $f w d^{P R O}$ are summarized in Fig. 3.

\section{Performance Evaluation}

We develop an OMNeT++ [9] based simulation framework and conduct extensive simulation experiments to evaluate the performance of the $f w d^{P R O}$. We consider a randomly generated network topology, where 5 data consumers, 5 data producers, and 20 to 40 routers are distributed. An exponential Interest packet rate with a mean value 1.0 is adopted by data consumers to request data content. The size of packet is 512 Bytes. The total simulation time is set to 500 seconds. In this paper, we consider interface utilization ratio, round-trip time (RTT), and NACK ratio as real-time network metrics to evaluate the overall performance of each outgoing interface alternative. The interface utilization ratio is calculated as the interface capacity divided by the amount of requested data but has not been received yet. We measure the performance in terms of Interest satisfaction ratio, Interest satisfaction latency, and average hop count. We also revisit prior schemes, BestRoute [7] and EPF [8], and modify them to work in the framework for performance comparison.

First, we measure the Interest satisfaction ratio against simulation time in Fig. 4, where the Interest satisfaction ratio is calculated as the total number of satisfied Interest packets divided by the total number of issued Interest packets. The BestRoute deliveries the lowest Interest satisfaction ratio because it adopts the coloring scheme with a single network metrics to make Interest packet forwarding decision. It is straightforward that the highest ranked Green outgoing interface might not be the best option when the Interest packet rate reaches the interface rate limit. In that case, the selected outgoing interface will experience significant traffic congestion when the Data packets come back. As a result, a number of Data packets will be dropped and a lower Interest satisfaction ratio will be observed. The EPF is an entropy-based probabilistic forwarding strategy to make a stochastic outgoing interface selection based on the working status of outgoing interface
Notations:

- $M, M^{\text {norm }}, x_{i j}, x_{i j}^{*}, M^{w g t}, w_{j}, x_{i j}^{\oplus}, S_{i}^{+}, S o l_{i}^{-}, \mathbb{I}_{i}$ : Defined before.

- $R_{k}$ and $I n_{j}^{\overleftarrow{j}}$ : A router $k$ and an outgoing interface $j$;

- $R T T_{k}[j]$ : A round-trip time of outgoing interface $j$ at router $k$;

- $N A C K_{k}[j]$ : A NACK ratio of outgoing interface $j$ at router $k$;

- $I U R_{k}[j]$ : An interface utilization ratio of outgoing interface $j$ at router $k$;

- $p k t[$ name,type]: A packet containing a name of data content (name) and packet type (type). Here, type can be either Interest, Data, or NACK.

$\diamond$ When $R_{k}$ receives a pkt [name, Data $]$ from $I n_{j}^{\leftarrow}$ : Update $R T T_{k}[j]$;

$\diamond$ When $R_{k}$ receives a $p k t[$ name, $N A C K]$ from $I n_{j}^{\leftarrow}$ : Update $N A C K_{k}[j]$;

$\diamond$ When $R_{k}$ forwards a $p k t[$ name, Interest $]$ through $I n_{j}^{\leftarrow}$ : Update $I U R_{k}[j]$;

$\diamond$ When $R_{k}$ has an Interest packet to forward:

Retrieve $R T T_{k}, N A C K_{k}$, and $I U R_{k}$; Create $M$;

Calculate $M^{\text {norm }}$ according to Eq. (1);

Calculate $M^{w g t}$ according to Eq. (2);

Calculate $\mathrm{Sol}^{+}$and $\mathrm{Sol}^{-}$according to Eq. (3) and (4);

Calculate $\mathbb{I}$ according to Eq. (5);

Rank all outgoing interface alternatives in terms of $\mathbb{I}$;

Select the highest ranked outgoing interface to forward Interest;

Fig. 3. The pseudocode of the proposed $f w d^{P R O}$ scheme.

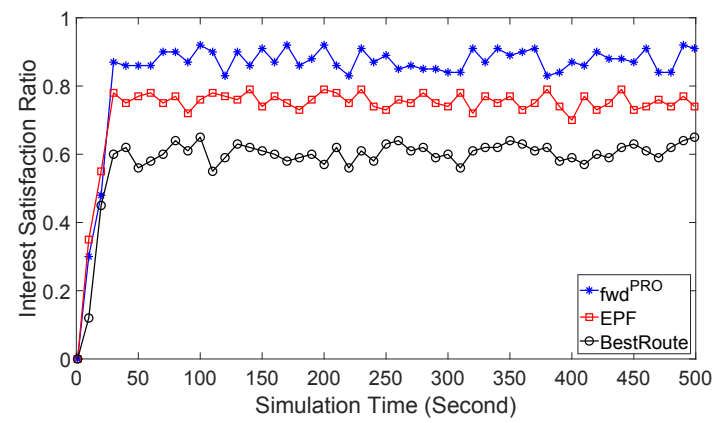

Fig. 4. Performance of Interest satisfaction ratio against simulation time.

and the smoothed round-trip time. The EPF obtains a higher Interest satisfaction ratio than BestRoute because it evaluates outgoing interface's static routing information and dynamic performance. By considering round-trip time, the EPF can easily detect the changes of network conditions, such as traffic congestion or packet lost, and selects a suitable outgoing interface to forward the Interest packet. Thus, more Data packets can be brought back to data consumers and a higher Interest satisfaction ratio is achieved. As shown in Fig. 4, it is obvious that the $f w d^{P R O}$ outperforms its two opponents in terms of Interest satisfaction ratio. This is because the $f w d^{P R O}$ employs TOPSIS to dynamically evaluate outgoing interface alternatives based on interface utilization ratio, round-trip time, and NACK ratio. Thus, the optimal outgoing interface can be selected to forward the Interest packet after balancing the tradeoff between multiple network metrics, and a larger number of Interest packets can be satisfied by Data packets.

Second, we obtain the average hop count and Interest satisfaction latency with a varying number of routers in the network 


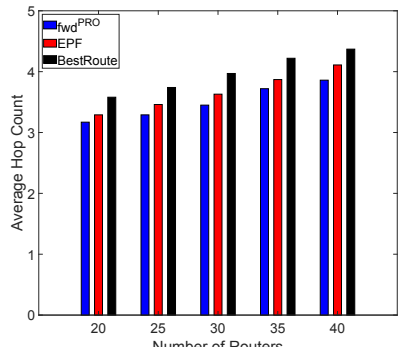

(a)

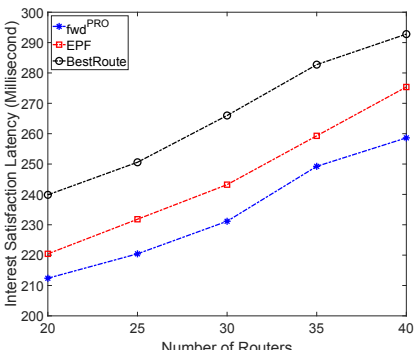

(b)
Fig. 5. Performance of average hop count and Interest satisfaction latency against number of routers.

in Fig. 5, where the number of routers is changed between 20 and 40. The average hop count is the average number of links a Data packet traverses to satisfy an issued Interest packet, while the Interest satisfaction latency is the amount of elapsed time from when the Interest packet is issued to when the Data packet is received. As shown in Fig. 5(a), a lower average hop count is obtained by $f w d^{P R O}$ and EPF because both of two schemes consider round-trip time as one of network metrics to select outgoing interface. If a longer round-trip time is observed on a certain outgoing interface, the reason can be either the downstream route experiences a packet lost or has more links to traverse. Thus, by selecting the outgoing interface with a smaller round-trip time, the Interest packet can be forwarded along a shorter route toward the data producer. Since the Data packet is transmitted along the reverse path of the Interest packet, thus, a lower average hop count is obtained by both $f w d^{P R O}$ and EPF. The $f w d^{P R O}$ still outperforms EPF because it evaluates the overall performance of outgoing interface with multiple network metrics, and a more reliable route can be chosen to transmit the Interest packet. It is shown in Fig. 5(b) that the $f w d^{P R O}$ can achieve the lowest Interest satisfaction latency compared to that of EPF and BestRoute. It is evident that the $f w d^{P R O}$ can bring Data packet along the shortest and most reliable route, thus, the lowest Interest satisfaction latency is achieved. The BestRoute shows the worst performance of Interest satisfaction latency because it does not consider round-trip time to select outgoing interface. Thus, it can end up choosing a longest route to transmit the Interest packet, as a result, the highest Interest satisfaction latency is obtained.

\section{Conclusion And Future Work}

In this paper, we proposed an adaptive forwarding strategy based on a multi-criteria decision-making model (TOPSIS) to realize intelligent and adaptive Interest packet forwarding in NDN. In the proposed forwarding strategy, each outgoing interface alternative is first evaluated based on multiple network metrics to obtain the forwarding index, which is an indicator of the overall performance. Then, all outgoing interface alternatives are ranked in terms of the forwarding index and the highest ranked one is chosen to forward the Interest packet. For performance evaluation, we considered interface utilization ratio, round-trip time (RTT), and NACK ratio as real-time network metrics. We also developed a cus- tomized discrete event driven simulation framework by using OMNeT++ and evaluated its performance through extensive simulation experiments. The simulation results indicate that the proposed approach is a viable Interest packet forwarding strategy in NDN. As a future work, we plan to investigate Analytic Network Process (ANP) to analyze the interrelationships between decision levels and multiple network metrics and dynamically calculate the relative weights of multiple network metrics. In addition, we plan to further extend the proposed scheme with the feature of Interest traffic load balancing. For example, the router can stochastically select an outgoing interface to forward the Interest packet by randomly generating a number and comparing it with the forwarding index of each outgoing interface. If the forwarding index of outgoing interface is larger than randomly generated number, this outgoing interface is chosen to forward the Interest packet. In this way, each outgoing interface will have a chance to forward the Interest packet, which can achieve the goal of traffic load balancing.

\section{REFERENCES}

[1] C. Pu, "Sybil Attack in RPL-Based Internet of Things: Analysis and Defenses," IEEE Internet of Things Journal (Early Access), pp. 1-1, 2020.

[2] C. Pu and L. Carpenter, "Psched: A Priority-Based Service Scheduling Scheme for the Internet of Drones," IEEE Systems Journal (Early Access), pp. 1-10, 2020.

[3] Cisco Annual Internet Report (2018-2023) White Paper, 2020.

[4] C. Pu, N. Payne, and J. Brown, "Self-Adjusting Share-Based Countermeasure to Interest Flooding Attack in Named Data Networking," in Proc. IEEE CPSCom, 2019, pp. 142-147.

[5] L. Zhang, A. Afanasyev, J. Burke, V. Jacobson, K. Claffy, P. Crowley, C. Papadopoulos, L. Wang, and B. Zhang, "Named Data Networking," ACM SIGCOMM Computer Communication Review, vol. 44, no. 3, pp. 66-73, 2014.

[6] Z. Li, Y. Xu, B. Zhang, L. Yan, and K. Liu, "Packet Forwarding in Named Data Networking Requirements and Survey of Solutions," IEEE Communications Surveys \& Tutorials, vol. 21, no. 2, pp. 1950-1987, 2018.

[7] C. Yi, A. Afanasyev, I. Moiseenko, L. Wang, B. Zhang, and L. Zhang, "A case for stateful forwarding plane," Computer Communications, vol. 36 , no. 7, pp. 779-791, 2013.

[8] K. Lei, J. Wang, and J. Yuan, "An Entropy-based Probabilistic Forwarding Strategy in Named Data Networking," in Proc. IEEE ICC, 2015, pp. $5665-5671$

[9] A. Varga, OMNeT++, 2014, http://www.omnetpp.org/.

[10] M. Abdelaal, M. Karadeniz, F. Dürr, and K. Rothermel, "liteNDN: QoSAware Packet Forwarding and Caching for Named Data Networks," in Proc. IEEE CCNC, 2020, pp. 1-9.

[11] A. Abane, M. Daoui, S. Bouzefrane, and P. Muhlethaler, "A lightweight forwarding strategy for Named Data Networking in low-end IoT," Journal of Network and Computer Applications, vol. 148, p. 102445, 2019.

[12] Y. Zhang, B. Bai, K. Xu, and K. Lei, "IFS-RL: An Intelligent Forwarding Strategy Based on Reinforcement Learning in Named-Data Networking," in Proc. ACM NetAI, 2018, pp. 54-59.

[13] P. Moll, J. Janda, and H. Hellwagner, "Adaptive Forwarding of Persistent Interests in Named Data Networking," in Proc. ACM ICN, 2017, pp. $180-181$.

[14] J. Yao, B. Yin, X. Tan, and X. Jiang, "A POMDP framework for forwarding mechanism in named data networking," Computer Networks, vol. 112, pp. 167-175, 2017.

[15] J. Lv, X. Tan, Y. Jin, and J. Zhu, "DRL-based Forwarding Strategy in Named Data Networking," in Proc. IEEE CCC, 2018, pp. 6493-6498.

[16] G. Tzeng and J. Huang, Multiple Attribute Decision Making: Methods and Applications. CRC press, 2011. 